\title{
What is the perioperative nurses main challenge when caring for the surgical patient?
}

\author{
Brid O' Brien
}

School of Nursing \& Midwifery, UCC

\section{Introduction}

This study looked to speak and listen to nurses working in the perioperative setting to identify and resolve their main challenges and concerns. Perioperative nursing is defined as the care of the patient before, during and immediately after surgery and has a rich history. Being at the frontline of care, the challenges and concerns nurses continually face in their daily working lives impact on the care they deliver to the patients in their care The nurses who participated in the study were all qualified and ranged from working in the perioperative setting from six months to thirty three years and were in both management and non-management positions.

\section{Background}

The aim of this study seeks to identify patterns of behaviour occurring daily in the perioperative setting. It seeks to identify the main concern of nurses working there and how they resolve issues that arise. Perioperative nursing can be defined as the provision of nursing care by a Registered Nurse preoperatively (before), intra-operatively (during), and postoperatively (after) to a patient undergoing an operative or invasive procedure. It is practiced today in many different areas, e.g. hospital operating rooms, interventional radiology suites, cardiac catheterization laboratory (room in a hospital where diagnostic imaging equipment is used to support the catherization procedures) surgeon offices, endoscopy suites, ambulatory surgery centres and emergency trauma units. Perioperative nursing is a complex and exciting specialty.

The Core Nursing Skills of Perioperative Patient Care involve: critical thinking, aseptic technique (procedure performed under sterile conditions), working with diverse patients and evidence based practice to ensure that quality care is delivered. It also involves maintaining safety, educating patients, adhering to legal guidelines, policies and procedures' ensuring that care is administered in an ethical way. The fundamental nursing values (knowledge, skills, and judgment) are ever present when working in the perioperative setting. These are the basis for quality care that surgical patients have relied on and 
can expect when being cared for by any perioperative nurse. Perioperative nursing is not just technical work that a nurse can do. A significant part of perioperative nursing is the delivery of scientifically based care which involves understanding the need for certain methods of care and when to initiate them. Knowledge of surgical interventions, instruments and equipment is required. Without this the perioperative nurse is unable to prepare for or anticipate the steps in the surgical procedure. It must be remembered that perioperative nursing is a systematic planned process. Many skills are required by perioperative nurses, i.e. nursing care skills (assessment), interpersonal skills, critical thinking and decision making and technical skills to ensure good quality care is given. Teamwork is fundamental in the operating room and occurs from the moment one steps inside the unit. Participation in a multidisciplinary team is part of working in the perioperative setting.

Normally the perioperative period consists of the preoperative, intraoperative and postoperative phase. The preoperative phase begins with the decision to have surgery until the patient is transferred to operating theatre. The preoperative period is the time period prior to surgery. The intraoperative period extends from admission to operating department to the transfer to recovery room. The postoperative period extends from the admission to recovery room to complete recovery from surgery. Surgery is normally classified under three headings: urgent (which implies elective or emergency), risk (which implies minor or major) and purpose (which implies diagnostic, removal, palliative, reconstructive, transplantation and constructive).

Recent reconfiguration changes within healthcare management in perioperative care have resulted in the movement of all major and minor surgery to the larger city hospitals. Contributing factors include government policy, major errors in diagnostic procedures, finance and creating centres of excellence.

\section{Method}

This study was guided by the principles of classical Grounded Theory, which is an an inductive and interpretative research methodology which creates research-based knowledge about behavioural pattern as described by Dr Barney Glaser. Following ethical approval, data was collected through individual, face to face, unstructured interviews with thirty seven perioperative nurses with varying lengths of experiences of working in the area. Thirty three hours of observation was also undertaken within the eight different perioperative settings within the Republic of Ireland. Theoretical sampling was utilised for the study which enabled making a decision in keeping with the emerging data to identify new participants to interview. Data was simultaneous collected and analysed according to established classic grounded theory research procedures of open and selective coding, constant comparison, memo-writing, and theoretical sampling.

The interviews were recorded and subsequently transcribed. The interviews lasted between twenty five minutes to ninety minutes. During the initial interviews the nurses 
were asked broad based open-ended questions regarding the challenges they face daily and they were listened to without verbal interruption, thus ensuring that the researcher remained open to the data. As the interviews continued, leads were followed and the questions became more focused while remaining open-ended. Following interviewing of the participants, the interviews were coded using substantive coding (open and selective) and constantly compared for similarities and differences. Memos were written in varying lengths recording ideas, developing concepts, identifying and theorising codes and their relationships and hypothesising about connections and relationships between categories. Memos were then sorted and theoretical coding was undertaken as per classical grounded theory approach.

This study is current and the core variable to emerge from the study was minimising risk in a high risk setting (main challenge facing nurses in the perioperative setting). This challenge was resolved by the perioperative nurses through "Anticipatory Vigilance". This is now being written up in an explanatory theory.

\section{Conclusion and Recommendations}

This study has provided the opportunity to nurses working in the perioperative setting to share their experiences on how challenges in their work environment are encountered and resolved. There has been a dearth of in-depth research within this area. The area of nurse perception of risk has been largely unexplored to date. The findings from this study will provide valuable insight and awareness for nurses working in the perioperative setting and provide a deeper understanding of the work undertaken by nurses working in this acute setting for those working outside the setting.

The theory identified in this study will be relevant to a number of people both within and outside the perioperative setting. It is hoped that this theory will support perioperative nurses with a clearer understanding of the work they do and how they do it. It will add to the body of knowledge already available. It will support staff in creating best practice guidelines ensuring that perioperative care is given further air time, resulting in the perioperative setting being a safer place to work . This will lead to establishing better quality care for all patients and nurses that spend time in the perioperative area.

It is anticipated that this explanatory theory will inform policy both locally and nationally in the future. It is current in adding to the body of knowledge on risk management and assessment. It will inform and influence practice and future understanding of nursing in the perioperative setting. Nurses working in the perioperative setting have an important role in contributing to positive patient outcomes within the surgical setting and will achieve that through influencing practice.

Brid O' Brien (RGN, OND, Cert in Operation Theatre Technique, BNS, PG. Dip, RNT, MSc) is a lecturer in the Department of Nursing and Midwifery at the University of Limerick and is also a 
student in the School of Nursing \& Midwifery Brookfield Health Sciences Complex UCC, under the supervision of Dr. Tom Andrews and Professor Eileen Savage 